OPEN ACCESS

Edited by:

Feng-Huei Lin,

National Taiwan University, Taiwan

Reviewed by:

Xin Zhao,

Hong Kong Polytechnic University,

Hong Kong SAR, China

Menekse Ermis Sen,

Terasaki Institute for Biomedical

Innovation, United States

*Correspondence:

Peng Zhang

peng.zhang@siat.ac.cn

Specialty section:

This article was submitted to

Tissue Engineering and Regenerative Medicine,

a section of the journal Frontiers in Bioengineering and

Biotechnology

Received: 25 August 2021

Accepted: 27 October 2021

Published: 12 November 2021

Citation:

Lin J, Sun AR, Li J, Yuan T, Cheng W, Ke L, Chen J, Sun W, Mi S and Zhang P (2021) A Three-Dimensional Co-Culture Model for Rheumatoid

Arthritis Pannus Tissue.

Front. Bioeng. Biotechnol. 9:764212.

doi: 10.3389/fbioe.2021.764212

\section{A Three-Dimensional Co-Culture Model for Rheumatoid Arthritis Pannus Tissue}

\author{
Jietao Lin ${ }^{1,2,3}$, Antonia RuJia Sun ${ }^{1,2,3}$, Jian Li $^{1,2,3}$, Tianying Yuan ${ }^{4}$, Wenxiang Cheng ${ }^{1,2,3}$, \\ Liqing $\mathrm{Ke}^{1,2,3}$, Jianhai Chen ${ }^{1,2,3}$, Wei Sun ${ }^{4}$, Shengli $\mathrm{Mi}^{4}$ and Peng Zhang ${ }^{1,2,3 *}$
}

${ }^{1}$ Center for Translational Medicine Research and Development, Shenzhen Institute of Advanced Technology, Chinese Academy of Science, Shenzhen, China, ${ }^{2}$ University of Chinese Academy of Sciences, Beijing, China, ${ }^{3}$ Shenzhen Engineering Research Center for Medical Bioactive Materials, Shenzhen, China, ${ }^{4}$ Biomanufacturing Center, Department of Mechanical Engineering, Tsinghua University, Beijing, China

Three-dimensional (3D) co-culture models have closer physiological cell composition and behavior than traditional 2D culture. They exhibit pharmacological effects like in vivo responses, and therefore serve as a high-throughput drug screening model to evaluate drug efficacy and safety in vitro. In this study, we created a 3D co-culture environment to mimic pathological characteristics of rheumatoid arthritis (RA) pannus tissue. 3D scaffold was constructed by bioprinting technology with synovial fibroblasts $(\mathrm{MH} 7 \mathrm{~A})$, vascular endothelial cells (EA.hy 926) and gelatin/alginate hydrogels. Cell viability was observed during 7-day culture and the proliferation rate of co-culture cells showed a stable increase stage. Cell-cell interactions were evaluated in the 3D printed scaffold and we found that spheroid size increased with time. TNF- $\alpha$ stimulated MH7A and EA.hy 926 in 3D pannus model showed higher vascular endothelial growth factor (VEGF) and angiopoietin (ANG) protein expression over time. For drug validation, methotrexate (MTX) was used to examine inhibition effects of angiogenesis in 3D pannus co-culture model. In conclusion, this 3D co-culture pannus model with biological characteristics may help the development of anti-RA drug research.

Keywords: rheumatoid arthritis, 3D bioprinting, tissue engineering, pannus tissue model, drug screening

\section{INTRODUCTION}

Joint is a dynamic tissue that supports us to move, but it may suffer destruction of bone and cartilage because of arthritis like RA. Due to the genetic factor or immune system disorder, synovial membrane in RA patients presents abnormal proliferation of synovial cells and migration of inflammatory cells (Deane et al., 2017). Synovial joint is usually rich in blood vessels, which is a unique manifestation of RA. New vessels and hyperplastic fibrous tissue contribute to angiogenic disorders and form a complex vascular tissue called pannus (Veale et al., 2017). Angiogenesis not only provides more means for the spread of inflammatory cytokines and the infiltration of leukocyte but aggravate the formation of pannus (Maruotti et al., 2006; Elshabrawy et al., 2015). RA pannus is an aggressive and invasive tissue with massive leukocyte infiltration, proliferative synovial membranes and neovascularization, which is directly responsible for cartilage destruction and bone erosion (Lee and Weinblatt, 2001). The development of pannus is highly relevant to the growth factors, pro-inflammatory cytokines and chemokines. Growth factors, such as vascular endothelial growth factor (VEGF) and basic fibroblast growth factor (bFGF) are described as the key regulators 
in proliferation, migration and vascular formation. Proinflammatory cytokines like tumor necrosis factor (TNF)- $\alpha$, interleukin (IL)-6, which provide inflammatory conditions in RA synovium, have direct and indirect effects on other cell types to produce pro-angiogenic factors (Semerano et al., 2011).

In recent years, RA have become the most common form of inflammatory arthritis. Patients need to rely on drugs for control as it is an incurable disease (Doan and Massarotti, 2005; Yu et al., 2018). Inhibition of angiogenesis can be a helpful strategy for early prevention and treatment of RA (Veale and Fearon, 2006; MacDonald et al., 2018; Balogh et al., 2019). However, RA drug testing has low accuracy and drug development cycle is long. Although animal model is the most effective way to study RA drugs before clinical trials, ethics and experimental accuracy limits rapid and efficient evaluation of drug safety and efficacy (Li and Izpisua Belmonte, 2019). To overcome these difficulties, co-culture models are often used to mimic physiological environment of pannus for RA study and anti-RA drug screening (D'andrea et al., 1998; Kasama et al., 2001; Nozaki et al., 2007; Chu et al., 2018; Gou et al., 2018). IBOLD et al. developed a $3 \mathrm{D}$ pannus model in vitro as a high-throughput screening assay. Chondrocytes from porcine donors were isolated and seeded them into wells to form extracellular matrix (ECM). After 14 days, it would be coated with human synovial fibroblasts. They found that intercellular communication between these 2 cell types occurs both through gap junctions and ATP-mediated paracrine stimulation. (D'andrea et al., 1998). In the co-culture model of chondrocytes and synovial cells, D'ANDREA et al. found that the $\mathrm{Ca}+$ signal between these 2 cell types can be affected by 18 a-glycyrrhetinic acid, suggesting they have communication in pannus tissue (Nozaki et al., 2007). Monocytes or polymorphonuclear neutrophils (PMNs) were seeded onto fibroblasts and Kasama et al. found that the expression of VEGF in co-culture groups are higher than synovial fibroblasts, monocytes or PMNs alone groups, which means VEGF expression in pannus can be also regulated by the interaction of synovial fibroblasts and activated leukocytes (Chu et al., 2018). Nozaki et al. isolated pannus tissue from RA patients and the inflammatory cells including macrophages, $\mathrm{T}$ cells and fibroblasts. They collected these cell types without enzyme digestion and found that inflammatory cells could develop into pannus-like tissue spontaneously in vitro. This pannus model continuously secreted MMP-9 and TNF- $\alpha$, IL-8 and M-CSF, which related with osteoclastogenesis (Gou et al., 2018). Although these studies revealed useful characteristics of $3 \mathrm{D}$ pannus models in vitro, it is still difficult to construct a long lasting and strong repeatability pannus model to test anti-RA drugs due to the limitations of fabrication techniques.

Recent advances in 3D fabrication technology have allowed direct assembly of cells and biocompatible materials to form in vitro cellular models for artificial organ regenerations, the study of disease mechanisms and drug screening. This promising technique has the advantages of accurate control of cell distribution, high simulation of physiological microenvironments and cost-effectiveness, which is suitable for constructing complex 3D in vitro models (Mandrycky et al., 2016; Ma et al., 2018; Ong et al., 2018; Zhu et al., 2020). Therefore, 3D printing has been applied in the establishments of disease pathogenesis and drug screening model in hepatocellular carcinoma (Sun et al., 2020; Xie et al., 2021), breast cancer (Swaminathan et al., 2019; Lv et al., 2021), cervical tumor (Zhao et al., 2014; Pang et al., 2018), bladder cancer (Kim et al., 2019), and neurodegenerative diseases (Thomas and Willerth, 2017). To apply the potential value of 3D printing on anti-RA drug research, in this paper we constructed the in vitro pannus model by $3 \mathrm{D}$ printing of endothelial cells (EA.hy 926)/Synovial fibroblasts (MH7A) and gelatin/alginate and characterized its biological function. To our knowledge, RA synovial tissue fibroblasts produce pro-angiogenic growth factors, cytokines under the induction of inflammatory mediators or hypoxia. Under the condition of pro-angiogenic and inflammatory factors, endothelial cells therefore promote cell proliferation, migration and tube formation (Szekanecz et al., 2005; Elshabrawy et al., 2015; Alam et al., 2017; Croft et al., 2019). Both MH7A cell line (synovial fibroblasts) and EA.hy 926 cell line (endothelial cells) are widely used to be the cell model in RA research as they are considered valuable in preclinical trials (Komorowski et al., 2006; Cheng et al., 2019; Qu et al., 2019; Kong et al., 2020). In addition, we used gelatin/alginate as they can mimics ECM to provide the cells a better natural microenvironment. They show good biocompatibility and good molding effect when building 3D biological scaffolds, and these structures could have long retention time (Sun et al., 2020; Lv et al., 2021). The schematic of 3D pannus scaffold printing process has been showed in Figure 1. Biological characterization of $3 \mathrm{D}$ printed pannus models on calcium cross-linking toxicity, cell proliferation, cell survival, cell morphology and VEGF and Angiopoietin (ANG) protein expression will be evaluated. Our findings may offer a basic view of $3 \mathrm{D}$ printed pannus model in drug screening application.

\section{MATERIALS AND METHODS}

\section{Cell Culture}

Human endothelial cells (EA.hy 926) were generously given by professor Qin's Laboratory and the cells were cultured in Dulbecco Modified Eagle Medium (DMEM) with $4.5 \mathrm{~g} / \mathrm{L}$ glucose, supplemented with $10 \%$ fetal bovine serum (Gibco, Thermo Fisher Scientific) and $1 \%$ penicillin/streptomycin (Sigma-Aldrich, MO, United States). Human synovial fibroblasts (MH7A) were purchased from the Riken Cell Bank (Tsukuba, Japan). The cells were maintained in Roswell Park Memorial Institute (RPMI) cultivation medium (Hyclone, Thermo Fisher Scientific, Wilmington, DE, United States) plus $10 \%$ fetal bovine serum (Gibco, Thermo Fisher Scientific) and 1\% penicillin/streptomycin (Sigma-Aldrich). For TNF-a co-culture model, EA.hy 926 and MH7A were pretreated with $20 \mathrm{ng} / \mathrm{ml}$ TNF- $\alpha$ for $6 \mathrm{~h}$ before printing. All culture experiments are under the condition of humidified air with $5 \% \mathrm{CO}_{2}$ in $37^{\circ} \mathrm{C}$.

\section{Bioink Preparation}

Gelatin/alginate was purchased from Sunp Biotech (Beijing, China). The lyophilized powder was dissolved in DMEM 


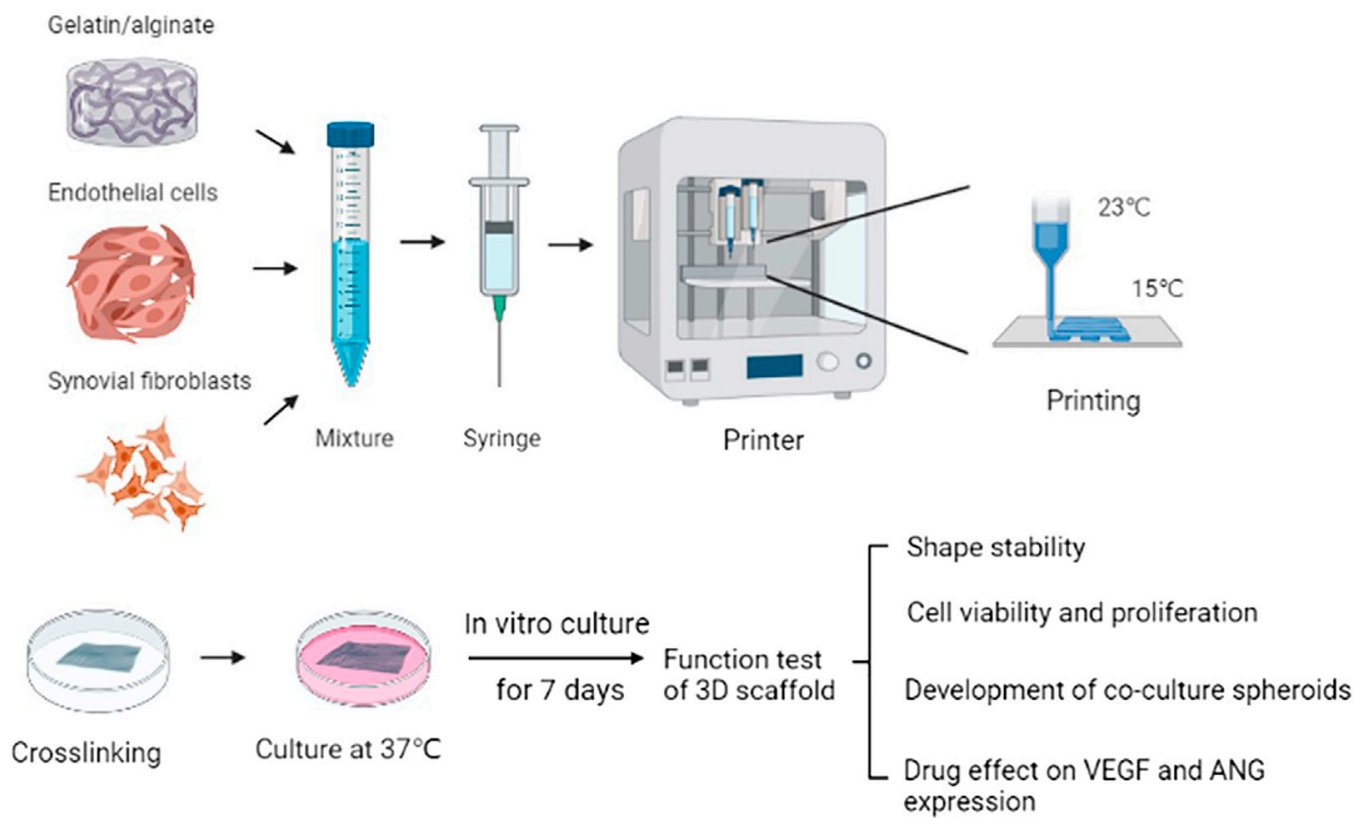

FIGURE 1 | Schematic of the 3D scaffold printing process. Fabrication of pannus mimic with gelatin /alginate and EA.hy 926/MH7A cells.

medium at $56^{\circ} \mathrm{C}$. The mixed ink should be pasteurized by $70^{\circ} \mathrm{C}$ for $30 \mathrm{~min}$ and $4^{\circ} \mathrm{C}$ for $10 \mathrm{~min}$ three times at constant temperature water bath and stored at $4^{\circ} \mathrm{C}$ until use. Before the experiment, the ink was kept at $37^{\circ} \mathrm{C}$ for $2 \mathrm{~h}$. For co-culture bioink, $2.5 \times 10^{6}$ cells of each EA.hy 926 and MH7A were resuspended in DMEM medium and homogeneously mixed with gelatin/alginate ink at a volume ratio at $1: 4$, resulting in a final cells density of $10^{6}$ cells $/ \mathrm{ml}$.

\section{D Scaffold Formation and Culture}

3D Cell Printer (SPP1603, SUNP, China) were used to fabricate all the $3 \mathrm{D}$ scaffold models. The temperature of the nozzle and printing bed were 23 and $15^{\circ} \mathrm{C}$, respectively. $25 \mathrm{G}$ needle was chosen and the scanning speed was controlled at $3 \mathrm{~mm} / \mathrm{s}$. The models were printed in an eight-layered square grid pattern with the size of $10 \times 10 \mathrm{~mm}$ cross sectional area and $2.4 \mathrm{~mm}$ thickness. The inks were loaded into $3 \mathrm{ml}$ printing syringe and precooled at the printing chamber for $10 \mathrm{~min}$. Preprinted on the $35 \mathrm{~mm}$ petri dishes and ensured that the bioink was smoothly extruded. After printing, hydrogel scaffolds were immersed in $\mathrm{CaCl}_{2}$ solution for 5 min for crosslinking with alginate, providing better strength to the scaffolds. All scaffolds were gently blown with a pipette to remove bubbles. Then the scaffolds were washed with sterile physiologic saline once and finally cultured in DMEM medium. The scaffolds were crosslinked and the medium was changed every 3 days.

\section{Calcium Cross-Linking Toxicity}

EA.hy 926, MH7A and co-culture mixed cells were seeded into 96-well culture plates $(3,000$ cells/well) for $24 \mathrm{~h}$. The cells were stimulated by $3 \% \mathrm{CaCl}_{2}$ solution for $5 \mathrm{~min}$ and washed by physiologic saline, and then they were cultured in DMEM medium for another 24 or $48 \mathrm{~h}$. To evaluate cellular metabolic activity, Cell counting kit-8 (CCK-8, Dojindo, Japan) were added into each well at the volume of $10 \%$ of the total, protected against exposure to light. After $3 \mathrm{~h}$ incubation at $37^{\circ} \mathrm{C}$, fluorescence of the culture medium was detected by microplate reader (PerkinElmer, Waltham, MA) at $450 \mathrm{~nm}$. The data was then normalized to the standard and calculated cell viability.

\section{Cell Proliferation Analysis}

Cell proliferation in printed scaffolds was studied using CCK-8 on cultured days $1,3,5$ and 7 . Cells were incubated in a mixture of culture medium and CCK- 8 for $2 \mathrm{~h}$. The values of fluorescence at $450 \mathrm{~nm}$ were compared among different printed groups.

\section{Cell Survival}

Cell survival test in 3D scaffolds was carried out on day 1, 3, 5, 7 after printing. Fluorescent Live/Dead assay (C2015M, Beyotime, China) was used according to the instruction manual. Briefly, medium was removed and the scaffolds were then washed twice with phosphate buffer solution (PBS). Subsequently, Calcein-AM and propidium iodide (PI) was mixed with detection buffer at the dilution ratio of 1:1,000 and 1.5:1,000, respectively. The cell laden scaffolds were incubated at $37^{\circ} \mathrm{C}$ for $30 \mathrm{~min}$ in dark, then washed three times with PBS. Calcein-AM marks viable cells green and propidium iodide (PI) shows dead cells red. Images were obtained from fluorescence microscopy (Leica, Germany).

\section{Cell Morphology Imaging and Analysis}

The scaffold shapes were taken with camera (Supplementary Figure S1). Two dimensional cell morphology of EA.hy 926 and MH7A was captured using inverted optical microscope (Carl Zeiss, Germany) at cell density of $80 \%$. Three-dimensional scaffold was examined by using fluorescence microscope (Leica, Germany) on cultured days 1, 3, 5 and 7. The images were taken in three random fields at $100 \times$ magnification. Cell diameters were measured by Image J (NIH, United States) software and analyzed by Origin (Originlab, United States). 


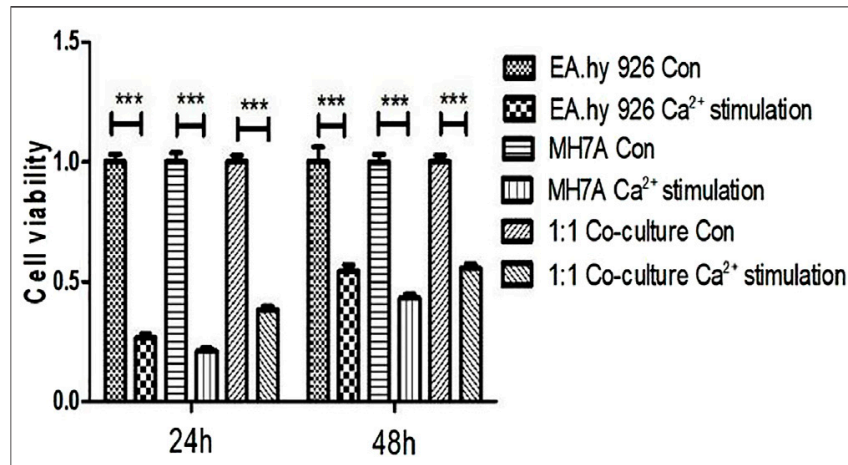

FIGURE 2|Cytotoxicity of calcium on EA. hy 926 and MH7A and 1:1 coculture mixture in 2D planar. Cells were treated with $\mathrm{CaCl} 2$ solution for 5 min and washed with physiologic saline once. After 24 and $48 \mathrm{~h}$, their viability was determined using CCK-8 assay (mean $\pm \mathrm{SD},{ }^{*} p<0.05 ;{ }^{* \star} p<0.01 ;{ }^{\star \star \star} p<$ 0.001 , t-test). Con $=$ no calcium stimulation; $S D=$ standard deviation.

\section{Enzyme-Linked Immunosorbent Assay}

The experiment was divided into four groups: blank (no cell) group, co-culture group, TNF- $\alpha$ pannus model group and TNF- $\alpha$ pannus treated by $100 \mathrm{nM}$ MTX for $24 \mathrm{~h}$ group. The concentrations of VEGF and ANG protein in the culture medium of 3D scaffold were detected on day 1, 3, 5, 7 . Secretory cytokines were examined using corresponding commercial ELISA kits (R\&D Systems, United States). A standard curve was constructed for each assay according to the manufacturer's instructions. The cytokine concentrations of each sample were calculated on the basis of the standard curve.

\section{Statistical Analysis}

All the data were presented as mean \pm SD. Statistical significance was evaluated by Students T Test. Differences were considered to be significant for $p<0.05 .{ }^{*} p<0.05 ;{ }^{* *} p<0.01$; ${ }^{* *} p<0.001$. Each experiment was performed in triplicate $(n=3)$ on at least independent three samples $(N \geq 3)$.

\section{RESULTS}

\section{Calcium Toxicity on Cells in 2D Planar Culture}

To test the cytotoxic effect of calcium on EA.hy 926 and MH7A and 1:1 co-culture mixture, CCK- 8 was used to determine the cell viability. As is shown in Figure 2, after stimulating by calcium for $5 \mathrm{~min}$ and cultured in DMEM for $24 \mathrm{~h}$, The percentage of EA.hy 926 cell viability decreased to $26.53 \pm 4.34$, but it rose to $54.49 \pm$ 6.17 at $48 \mathrm{~h}$ MH7A cells also had the same trend, $21.05 \pm 3.30$ percent at $24 \mathrm{~h}$ and $43.01 \pm 4.24$ percent at $48 \mathrm{~h}$, respectively. In terms of 1:1 co-culture mixture, they performed $38.29 \pm 3.71$ at $24 \mathrm{~h}$ and $55.62 \pm 4.17$ at $48 \mathrm{~h}$.

\section{Cell Distribution and Viability in 3D Scaffolds}

To determine cell distribution and survival in 3D gelatin/alginate/ EA.hy 926/MH7A model, we used calcein-AM/PI staining assay to analyze live/dead cells on day 1, 3, 5 and 7. As we can see, the cells were evenly distributed in gelatin/alginate scaffold. Cell viability was stable about $80 \%$ during the in vitro culture of EA.hy 926/MH7A in 3D scaffold (Figure 3A). The cellular proliferation in 3D scaffolds was detected using CCK-8 kit on the same time. Figure 3B demonstrates that compared with day 1, cells had 1.36-fold proliferation on day 3, 1.75-fold proliferation on day 5 , and 2.03 -fold proliferation on day 7 . There were significant differences between day $1(0.39 \pm 0.12)$ and day 5 $(0.68 \pm 0.05)$ and $7(0.79 \pm 0.05)$. Overall, the proliferation rate of co-culture cells had a stable increase stage from day 1 to day 7 .

\section{Development of Co-Culture Spheroids Within Cell Laden Scaffold}

Inverted optical microscope was used to observe the cell morphology in 2D planar culture. EA.hy 926 shows epithelioid morphology and MH7A shows epithelioid and polygonal morphology (Figure 4A). The cells and cellular distribution pattern in printed scaffolds were characterized using fluorescence microscope on day $1,3,5,7$. Compared with 2D planar culture, cells turned to be spheroids within 3D scaffolds, and they were observed to form larger spheroids after 3 days of bioprinting. The spheroid size increased over time. Figure 4B shows spheroid distribution in the 3D co-culture cell laden scaffold. At day 1, 7.09\% of the spheroids were at the diameter range of $20-30 \mu \mathrm{m}$. At day 3 , the size between $20-30 \mu \mathrm{m}$ was up to $28 \%$. The percentages of over $30 \mu \mathrm{m}$ at day 5 and day 7 were 10.65 and $14.73 \%$, respectively (Figure $4 \mathrm{C}$ ).

\section{Effect of 3D Engineered Scaffold on VEGF and ANG Expression}

The experiment was divided into four groups: blank (no cell) group, co-culture group, TNF- $\alpha$ pannus model group and TNF- $\alpha$ pannus treated by MTX group. The culture medium of the threedimensional scaffold was collected on day 1, 3, 5, 7. Figure 5A shows that the cell co-culture scaffold and pannus scaffold secreted more VEGF protein on day 5 and 7 compared with that on the first day, but there was no significant difference compared with the no cell group on the same day $(p>0.05)$. Figure 5B illustrates the content of ANG secreted protein in the co-culture scaffold on day 1 was different from that of the no cell group $(p<0.05)$, and there was a significant difference from day 3 to day $7(p<0.001)$. On the day 7 , the ANG protein concentration of the TNF- $\alpha$ pannus model group was different from that of the blank group $(p<0.05)$. Although the ANG concentration decreased in the pannus MTX group, there was no significant difference when compared with that in TNF- $\alpha$ pannus group.

\section{DISCUSSION}

RA is a chronic and systemic autoimmune disease, and immune dysregulation occurs earlier than joint inflammation. Current medications for RA are glucocorticoids (GCs), nonsteroidal antiinflammatory drugs (NSAIDs), disease-modifying antirheumatic drugs (DMARDs) and biological therapies, which provide clinically meaningful pain relief and control inflammation in 


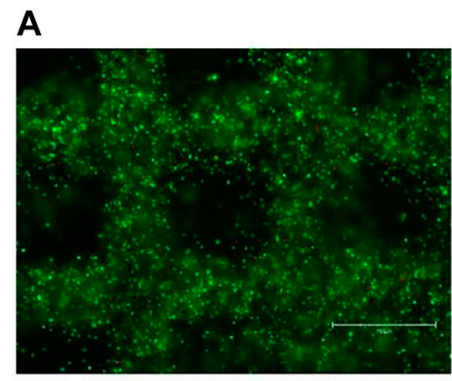

Day1

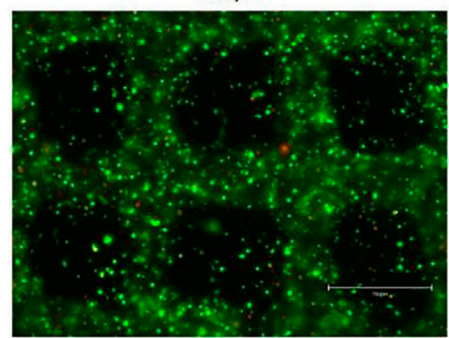

Day5

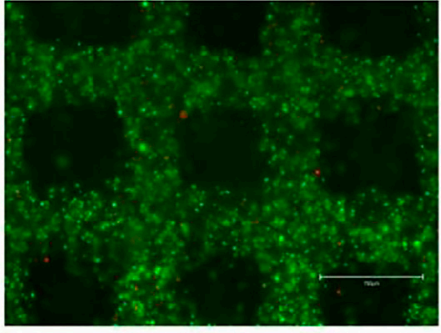

Day3

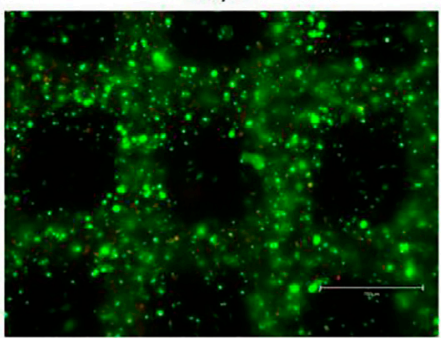

Day7
B

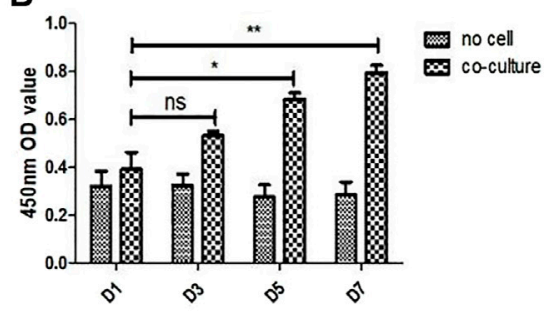

FIGURE 3 | Cell survival and proliferation in the 3D cell laden pannus tissue model. (A) Cell survival at different time points after printing. Live and dead cells were labelled with calcein-AM (green) and PI (red), respectively. Scale bar, $750 \mu \mathrm{m}$. (B) Proliferation rates of cells in 3D co-culture cell laden scaffolds at day 1, 3, 5, and 7.

\section{A}

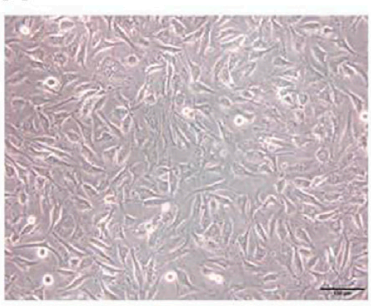

Endothelial cells (Ea.hy926)

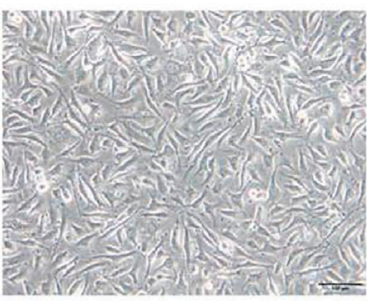

Synovial fibroblasts (MH7A)

B

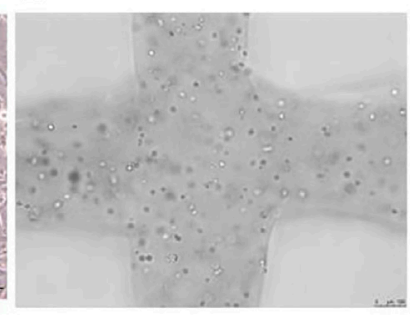

Day1

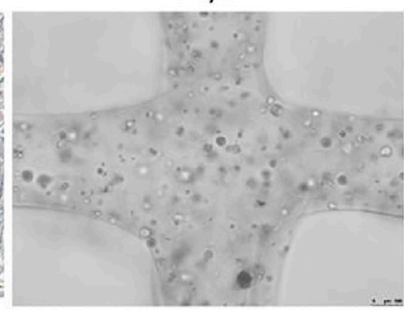

Day5

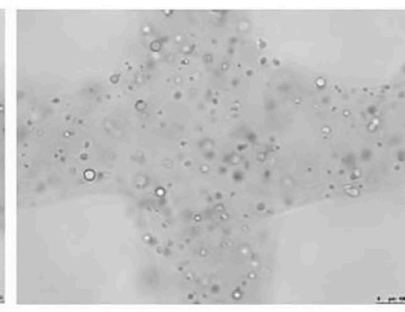

Day3

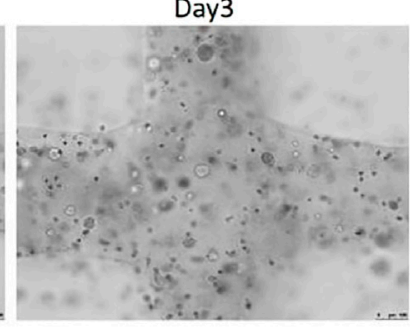

Day7
C

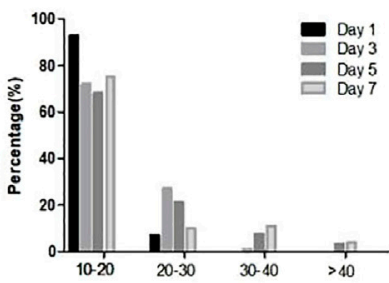

FIGURE 4 | Cellular morphological differences between 2D planar culture and 3D scaffolds. (A) EA.hy 926 and MH7A cells morphology in 2D planar culture. EA.hy 926 cells look epithelioid and MH7A cells are epithelioid and polygonal. Scale bar, $100 \mu \mathrm{m}$. (B) 3D Gelatin /alginate/EA.hy 926/MH7A scaffolds observed by a fluorescence microscope on culture days 1, 3, 5, and 7. Scale bar, $100 \mu \mathrm{m}$. Black arrows indicate cells and cellular spheroids in 3D scaffolds. (C) Distribution of spheroid diameter in 3D cell laden scaffolds on day $1,3,5$, and 7.

patients. However, several side effects, including cytopenia, psoriasis, lung disease and liver damage, have been proved in treatments with these drugs (Burmester and Pope, 2017; Abbasi et al., 2019). Thus, the discovery of a safe and effective drug for RA treatment remains a crucial challenge.
Over the last 2 decades, angiogenesis has been reported to play an important role in the deterioration of RA. RA pannus is an aggressive and invasive tissue with rich proinflammatory cytokines like TNF- $\alpha$, IL- $1 \beta$ and IL-6, which is directly responsible for cartilage destruction and bone erosion (Lee and Weinblatt, 

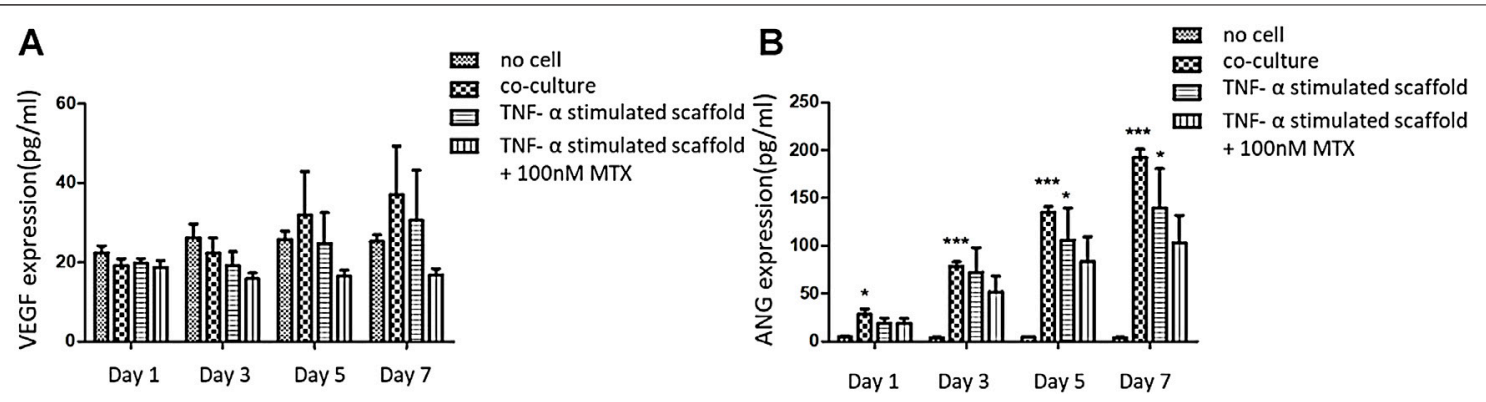

FIGURE 5 | Concentration of VEGF and ANG secreted protein in the supernatant of the three-dimensional scaffold on Day 1, 3, 5, and 7. (A) A. VEGF secreted protein concentration ( $\mathrm{pg} / \mathrm{ml})$; (B) ANG secreted protein concentration (pg/ml).

2001; MacDonald et al., 2018). Numerous researchers have studied on inhibition of angiogenesis in RA (Zhang et al., 2017; Yang et al., 2018; Li et al., 2019; Zhai et al., 2019). In order to screen anti-RA drugs in a more accurate and efficient way, here we developed a 3D printed model to mimic the microenvironment of RA pannus. In terms of cell composition, we chose vascular endothelial cells and synovial fibroblast. Since EA.hy 926 and MH7A cells are widely used in drug discovery in RA and they are cell lines, we chose them to ensure the effectiveness and repeatability of our model. However, single cell model failed to offer a complex microenvironment to evaluate cellular response to drugs accurately (Sun et al., 2019). In the microenvironment, we added inflammatory factors (TNF- $\alpha$ ) to simulate the inflammatory microenvironment. Compared to monolayer cell culture, 3D printing model is close to the space environment of the actual RA pannus tissue, enhancing the communication between cells and thereby improving the accuracy and efficiency of drug detection. For example, Kim et al. showed that cells grown in $2 \mathrm{D}$ culture conditions exhibit different gene and protein expression from those observed in vivo. And it was confirmed that cell proliferation rates in $3 \mathrm{D}$ culture was higher than that in the $2 \mathrm{D}$ cell culture because they mimic cell-to-cell interaction. They also found that there was a different anticancer effect between these two models. The drug effect in the $2 \mathrm{D}$ cell culture model is exaggerated, which explains why immunotherapies have shown excellent efficacy in research studies but not in clinical studies and patients (Kim et al., 2019). Thus, in this study we constructed the 3D pannus model based on the co-culture system of MH7A and EA.hy 926 cells. Alginate and gelatin are natural biomaterials. Both of them have high printability and good biocompatibility with cells. In addition, micro-extrusion printing has been shown that they can print cell laden scaffold in a controllable way with high cell viability. Despite cells experience stress condition during printing, they still have good cell viability with hydrogel (Mandrycky et al., 2016; Panwar and Tan, 2016). To maintain sufficient mechanical strength, printed scaffold needs to crosslink with $\mathrm{CaCl}_{2}$ solution every 3 days. Our result showed that $\mathrm{CaCl}_{2}$ solution had a cytotoxic effect among EA.hy 926 cells, MH7A cells, and 1:1 co-culture mixture groups in $2 \mathrm{D}$ planar. Cell viability decreased to around $30 \%$ after calcium stimulation for $5 \mathrm{~min}$ at $24 \mathrm{~h}$, but cell viability regulated to around half at $48 \mathrm{~h}$. According to the manufacturer's instruction, the recommendation time for crosslinking is $4-8 \mathrm{~min}$, and we chose $5 \mathrm{~min}$ in order to hold the shape of the scaffold. As the concentration and ratio of gelatin and sodium alginate are confidential and there would be difference between 2D culture and 3D hydrogel scaffold, we further tested cell survival using calcein-AM/PI staining assay and measured cell proliferation using CCK- 8 assay in $3 \mathrm{D}$ cell laden scaffold. The survival result showed that the viability of the co-culture mixture was around $80 \%$. The proliferation rate of co-culture cells increased steadily from day 1 to day 7 , which illustrates the gelatin/alginate bioink is biocompatible with cell growth. Earlier studies have demonstrated that cell viability and behavior in the scaffold is influenced by biomaterial type, material viscosity, printing speed, printing temperature and extrusion pressure (Zhao et al., 2015; Li et al., 2018). During our exploration on printing process, we noticed that too low temperature would lead to over coagulation of bioink. The bioink was so difficult to extrude that shear forces increased, resulting in cellular injury and death.

Compared with the morphology of MH7A cells and EA.hy 926 in $2 \mathrm{D}$ planar culture and $3 \mathrm{D}$ printed scaffold, we found that MH7A showed epithelioid and polygonal morphology and EA.hy 926 presented epithelioid morphology while they looked spheroids from day 1 to day 7 . What's more, after we measured the diameter of cellular spheroids on day 1, 3, 5 and 7, we observed that cells were assembled to be larger spheroid with time.

VEGF and ANG are considered fundamental in the formation of pannus. VEGF is one of the key regulators of angiogenesis as they are related to proliferation, migration and vascular tube formation (Marrelli et al., 2011). ANG acts later in the pathogenesis of pannus compared to VEGF. ANG is increased to form and increase blood vessel stability (Clavel et al., 2003). In order to verify the biological function of our 3D printed scaffold, we conducted ELISA assay to detect concentrations of VEGF and ANG protein. The results showed that the expression of VEGF and ANG in the co-culture and pannus group increased with days, but the co-culture group showed a more obvious effect. This may be because the cells in pannus group was induced by TNF- $\alpha$ before the model was constructed, but we did not provide an external inflammatory environment afterwards, resulting in the incomplete performance of the pannus characteristics. MTX has been showed that it could reduce VEGF content in CIA rat model to relieve angiogenesis (Chen et al., 2021). Hirata S also illustrated that MTX inhibited both basal and vascular endothelial cell growth 
factor-stimulated tritiated deoxyuridine (3H-UdR) incorporation into vascular endothelial cell in a dose-dependent manner (Hirata et al., 1989). So MTX was added after the model was printed, and the concentration of pro-angiogenic factors decreased compared with the pannus group for 7 days.

However, there are some differences between our 3D pannus scaffold and the actual pannus tissue. Here we only chose vascular endothelial cells and synovial fibroblast to mimic the cell composition of RA pathological pannus tissue instead of using all cell types. The pro-angiogenic factors in vivo, such as growth factors, hypoxia inducible factors, cytokines, chemokines, matrix metalloproteinase and adhesion molecules are also complex in deterioration of pannus. In this study, we constructed a 3D coculture model for RA pannus tissue and provided a basic view of its biological characteristics. Further work on the comparison of pathological characteristics between pannus model of RA in vitro and clinical pannus specimens is needed, and improvements of this scaffold should be processed in the future.

\section{CONCLUSION}

We report the construction of in vitro RA pannus co-culture model by applying 3D printing technique with EA.hy 926/MH7A and gelatin/alginate. The 3D pannus model showed a good cell viability and interaction to mimic the microenvironment of pannus in vivo. The concentration of VEGF and ANG protein in the supernatant of the 3D pannus model increased over time. In addition, adding MTX to the 3D pannus model can downregulate the expression of pro-angiogenic factors. Further studies are required to develop more details to construct the platform for drug screening, but this study may offer a basic view of 3D printed pannus model in drug screening application.

\section{DATA AVAILABILITY STATEMENT}

The raw data supporting the conclusion of this article will be made available by the authors, without undue reservation.

\section{REFERENCES}

Abbasi, M., Mousavi, M. J., Jamalzehi, S., Alimohammadi, R., Bezvan, M. H., Mohammadi, H., et al. (2019). Strategies toward Rheumatoid Arthritis Therapy; the Old and the New. J. Cell. Physiol. 234 (7), 10018-10031. doi:10.1002/jcp.27860

Alam, J., Jantan, I., and Bukhari, S. (2017). Rheumatoid Arthritis: Recent Advances on its Etiology, Role of Cytokines and Pharmacotherapy. Biomed. Pharmacother. 92, 615-633. doi:10.1016/j.biopha.2017.05.055

Balogh, E., Biniecka, M., Fearon, U., Veale, D. J., and Szekanecz, Z. (2019). Angiogenesis in Inflammatory Arthritis. The Isr. Med. Assoc. J. 21 (5), 345-352.

Burmester, G. R., and Pope, J. E. (2017). Novel Treatment Strategies in Rheumatoid Arthritis. Lancet (London, England) 389 (10086), 2338-2348. doi:10.1016/ S0140-6736(17)31491-5

Chen, J., Cheng, W., Li, J., Wang, Y., Chen, J., Shen, X., et al. (2021). Notch-1 and Notch3 Mediate Hypoxia-Induced Activation of Synovial Fibroblasts in Rheumatoid Arthritis. Arthritis Rheumatol. 73 (10), 1810-1819. doi:10.1002/art.41748

\section{AUTHOR CONTRIBUTIONS}

JtL, AS, JnL, WC, and PZ contributed to the conception of the study. JnL, TY, LK, and JC conducted the experiment. SM and WS provided the $3 \mathrm{D}$ printing machine. JtL, JnL, and JC performed the data analyses and wrote the manuscript. PZ supervised and trained JtL and he has been the corresponding author for the manuscript.

\section{FUNDING}

This research was supported by the National Key R\&D Program of China, China: 2018YFC1705205; Foreign cooperation project of Chinese Academy of Sciences, China: 172644KYSB20190032; National Natural Science Foundation of China, China: 92068117 and 81871809; Guangdong Basic and Applied Basic Research Fund, China: 2020B1515120052; Sanming Project of Medicine in Shenzhen, China: SZSM201808072; Development and Reform Commission of Shenzhen Municipality, China: XMHT20190106001. Shenzhen Double Chain Project for Innovation and Development Industry supported by Bureau of Industry and Information Technology of Shenzhen, China: 201908141541. The flow chart of 3D scaffold printing process was made on Biorender.com.

\section{ACKNOWLEDGMENTS}

Authors also wish to acknowledge the great help from Prof. Mi and Prof. Sun of Tsinghua University.

\section{SUPPLEMENTARY MATERIAL}

The Supplementary Material for this article can be found online at: https://www.frontiersin.org/articles/10.3389/fbioe.2021.764212/ full\#supplementary-material

Cheng, W. X., Huang, H., Chen, J. H., Zhang, T. T., Zhu, G. Y., Zheng, Z. T., et al. (2019). Genistein Inhibits Angiogenesis Developed during Rheumatoid Arthritis through the IL-6/JAK2/STAT3/VEGF Signalling Pathway. J. orthopaedic translation 22, 92-100. doi:10.1016/j.jot.2019.07.007

Chu, J., Wang, X., Bi, H., Li, L., Ren, M., and Wang, J. (2018). Dihydromyricetin Relieves Rheumatoid Arthritis Symptoms and Suppresses Expression of Proinflammatory Cytokines via the Activation of Nrf2 Pathway in Rheumatoid Arthritis Model. Int. immunopharmacol 59, 174-180. doi:10.1016/ j.intimp.2018.04.001

Clavel, G., Bessis, N., and Boissier, M. C. (2003). Recent Data on the Role for Angiogenesis in Rheumatoid Arthritis. Jt. bone Spine. 70 (5), 321-326. doi:10.1016/s1297-319x(03)00088-5

Croft, A. P., Campos, J., Jansen, K., Turner, J. D., Marshall, J., Attar, M., et al. (2019). Distinct Fibroblast Subsets Drive Inflammation and Damage in Arthritis. Nature 570 (7760), 246-251. doi:10.1038/ s41586-019-1263-7 
D'andrea, P., Calabrese, A., and Grandolfo, M. (1998). Intercellular Calcium Signalling between Chondrocytes and Synovial Cells in Co-culture. Biochem. J. 329 (Pt 3), 681-687. doi:10.1042/bj3290681

Deane, K. D., Demoruelle, M. K., Kelmenson, L. B., Kuhn, K. A., Norris, J. M., and Holers, V. M. (2017). Genetic and Environmental Risk Factors for Rheumatoid Arthritis. Best Practice \& Research. Clin. Rheumatol. 31 (1), 3-18. doi:10.1016/ j.berh.2017.08.003

Doan, T., and Massarotti, E. (2005). Rheumatoid Arthritis: an Overview of New and Emerging Therapies. J. Clin. Pharmacol. 45 (7), 751-762. doi:10.1177/ 0091270005277938

Elshabrawy, H. A., Chen, Z., Volin, M. V., Ravella, S., Virupannavar, S., and Shahrara, S. (2015). The Pathogenic Role of Angiogenesis in Rheumatoid Arthritis. Angiogenesis 18 (4), 433-448. doi:10.1007/s10456-015-9477-2

Gou, K. J., Zeng, R., Ren, X. D., Dou, Q. L., Yang, Q. B., Dong, Y., et al. (2018). Antirheumatoid Arthritis Effects in Adjuvant-Induced Arthritis in Rats and Molecular Docking Studies of Polygonum Orientale L. Extracts. Immunol. Lett. 201, 59-69. doi:10.1016/j.imlet.2018.11.009

Hirata, S., Matsubara, T., Saura, R., Tateishi, H., and Hirohata, K. (1989). Inhibition of In Vitro Vascular Endothelial Cell Proliferation and In Vivo Neovascularization by Low-Dose Methotrexate. Arthritis Rheum. 32 (9), 1065-1073. doi:10.1002/anr.1780320903

Kasama, T., Shiozawa, F., Kobayashi, K., Yajima, N., Hanyuda, M., Takeuchi, H. T., et al. (2001). Vascular Endothelial Growth Factor Expression by Activated Synovial Leukocytes in Rheumatoid Arthritis: Critical Involvement of the Interaction with Synovial Fibroblasts. Arthritis Rheum. 44 (11), 2512-2524. doi:10.1002/1529-0131(200111)44:11<2512:aid-art431>3.0.co;2-o

Kim, M. J., Chi, B. H., Yoo, J. J., Ju, Y. M., Whang, Y. M., and Chang, I. H. (2019). Structure Establishment of Three-Dimensional (3D) Cell Culture Printing Model for Bladder Cancer. PloS one 14 (10), e0223689. doi:10.1371/ journal.pone.0223689

Komorowski, J., Jerczyńska, H., Siejka, A., Barańska, P., Ławnicka, H., Pawłowska, Z., et al. (2006). Effect of Thalidomide Affecting VEGF Secretion, Cell Migration, Adhesion and Capillary Tube Formation of Human Endothelial EA.Hy 926 Cells. Life Sci. 78 (22), 2558-2563. doi:10.1016/j.lfs.2005.10.016

Kong, L., Wang, L., Zhao, Q., Di, G., and Wu, H. (2020). Rhodojaponin II Inhibits TNF- $\alpha$-Induced Inflammatory Cytokine Secretion in MH7A Human Rheumatoid Arthritis Fibroblast-like Synoviocytes. J. Biochem. Mol. Toxicol. 34 (10), e22551. doi:10.1002/jbt.22551

Lee, D. M., and Weinblatt, M. E. (2001). Rheumatoid Arthritis. Lancet (London, England) 358 (9285), 903-911. doi:10.1016/S0140-6736(01)06075-5

Li, M., and Izpisua Belmonte, J. C. (2019). Organoids - Preclinical Models of Human Disease. New Engl. J. Med. 380 (6), 569-579. doi:10.1056/NEJMra1806175

Li, Y., Yang, B., Bai, J. Y., Xia, S., Mao, M., Li, X., et al. (2019). The Roles of Synovial Hyperplasia, Angiogenesis and Osteoclastogenesis in the Protective Effect of Apigenin on Collagen-Induced Arthritis. Int. immunopharmacol. 73, 362-369. doi:10.1016/j.intimp.2019.05.024

Li, Z., Huang, S., Liu, Y., Yao, B., Hu, T., Shi, H., et al. (2018). Tuning AlginateGelatin Bioink Properties by Varying Solvent and Their Impact on Stem Cell Behavior. Scientific Rep. 8 (1), 8020. doi:10.1038/s41598-018-26407-3

Lv, K., Zhu, J., Zheng, S., Jiao, Z., Nie, Y., Song, F., et al. (2021). Evaluation of Inhibitory Effects of Geniposide on a Tumor Model of Human Breast Cancer Based on 3D Printed Cs/Gel Hybrid Scaffold. Mater. Sci. Eng. C, Mater. Biol. Appl. 119, 111509. doi:10.1016/j.msec.2020.111509

Ma, X., Liu, J., Zhu, W., Tang, M., Lawrence, N., Yu, C., et al. (2018). 3D Bioprinting of Functional Tissue Models for Personalized Drug Screening and In Vitro Disease Modeling. Adv. Drug Deliv. Rev. 132, 235-251. doi:10.1016/j.addr.2018.06.011

MacDonald, I. J., Liu, S. C., Su, C. M., Wang, Y. H., Tsai, C. H., and Tang, C. H. (2018). Implications of Angiogenesis Involvement in Arthritis. Int. J. Mol. Sci. 19 (7), 2012. doi:10.3390/ijms19072012

Mandrycky, C., Wang, Z., Kim, K., and Kim, D. H. (2016). 3D Bioprinting for Engineering Complex Tissues. Biotechnol. Adv. 34 (4), 422-434. doi:10.1016/ j.biotechadv.2015.12.011

Marrelli, A., Cipriani, P., Liakouli, V., Carubbi, F., Perricone, C., Perricone, R., et al. (2011). Angiogenesis in Rheumatoid Arthritis: a Disease Specific Process or a Common Response to Chronic Inflammation? Autoimmun. Rev. 10 (10), 595-598. doi:10.1016/j.autrev.2011.04.020
Maruotti, N., Cantatore, F. P., Crivellato, E., Vacca, A., and Ribatti, D. (2006). Angiogenesis in Rheumatoid Arthritis. Histology and histopathol 21 (5), 557-566. doi:10.14670/HH-21.557

Nozaki, T., Takahashi, K., Ishii, O., Endo, S., Hioki, K., Mori, T., et al. (2007). Development of an Ex Vivo Cellular Model of Rheumatoid Arthritis: Critical Role of CD14-Positive Monocyte/macrophages in the Development of Pannus Tissue. Arthritis Rheum. 56 (9), 2875-2885. doi:10.1002/art.22849

Ong, C. S., Yesantharao, P., Huang, C. Y., Mattson, G., Boktor, J., Fukunishi, T., et al. (2018). 3D Bioprinting Using Stem Cells. Pediatr. Res. 83 (1-2), 223-231. doi:10.1038/pr.2017.252

Pang, Y., Mao, S. S., Yao, R., He, J. Y., Zhou, Z. Z., Feng, L., et al. (2018). TGF- $\beta$ Induced Epithelial-Mesenchymal Transition in an Advanced Cervical Tumor Model by 3D Printing. Biofabrication 10 (4), 044102. doi:10.1088/1758-5090/aadbde

Panwar, A., and Tan, L. P. (2016). Current Status of Bioinks for Micro-extrusionbased 3D Bioprinting. Molecules (Basel, Switzerland) 21 (6), 685. doi:10.3390/ molecules 21060685

Qu, S. P., Li, G. W., Ma, H., and Xing, Q. (2019). MicroRNA-193a-3p Participates in the Progression of Rheumatoid Arthritis by Regulating Proliferation and Apoptosis of MH7A Cells through Targeting IGFBP5. Eur. Rev. Med. Pharmacol. Sci. 23 (11), 4850-4857. doi:10.26355/eurrev_201906_18072

Semerano, L., Clavel, G., Assier, E., Denys, A., and Boissier, M. C. (2011). Blood Vessels, a Potential Therapeutic Target in Rheumatoid Arthritis? Jt. bone Spine 78 (2), 118-123. doi:10.1016/j.jbspin.2010.06.004

Sun, L., Yang, H., Wang, Y., Zhang, X., Jin, B., Xie, F., et al. (2020). Application of a 3D Bioprinted Hepatocellular Carcinoma Cell Model in Antitumor Drug Research. Front. Oncol. 10, 878. doi:10.3389/fonc.2020.00878

Sun, W., Luo, Z., Lee, J., Kim, H. J., Lee, K., Tebon, P., et al. (2019). Organ-on-aChip for Cancer and Immune Organs Modeling. Adv. Healthc. Mater. 8 (15), e1900754. doi:10.1002/adhm.201900754

Swaminathan, S., Hamid, Q., Sun, W., and Clyne, A. M. (2019). Bioprinting of 3D Breast Epithelial Spheroids for Human Cancer Models. Biofabrication 11 (2), 025003. doi:10.1088/1758-5090/aafc49

Szekanecz, Z., Gáspár, L., and Koch, A. E. (2005). Angiogenesis in Rheumatoid Arthritis. Front. Biosci. 10, 1739-1753. doi:10.2741/1657

Thomas, M., and Willerth, S. M. (2017). 3-D Bioprinting of Neural Tissue for Applications in Cell Therapy and Drug Screening. Front. Bioeng. Biotechnol. 5, 69. doi:10.3389/fbioe.2017.00069

Veale, D. J., and Fearon, U. (2006). Inhibition of Angiogenic Pathways in Rheumatoid Arthritis: Potential for Therapeutic Targeting. Best Practice \& Research. Clin. Rheumatol. 20 (5), 941-947. doi:10.1016/j.berh.2006.05.004

Veale, D. J., Orr, C., and Fearon, U. (2017). Cellular and Molecular Perspectives in Rheumatoid Arthritis. Semin. immunopathol 39 (4), 343-354. doi:10.1007/ s00281-017-0633-1

Xie, F., Sun, L., Pang, Y., Xu, G., Jin, B., Xu, H., et al. (2021). Three-dimensional Bio-Printing of Primary Human Hepatocellular Carcinoma for Personalized Medicine. Biomaterials 265, 120416. doi:10.1016/j.biomaterials.2020.120416

Yang, G., Chang, C. C., Yang, Y., Yuan, L., Xu, L., Ho, C. T., et al. (2018). Resveratrol Alleviates Rheumatoid Arthritis via Reducing ROS and Inflammation, Inhibiting MAPK Signaling Pathways, and Suppressing Angiogenesis. J. Agric. Food Chem. 66 (49), 12953-12960. doi:10.1021/acs.jafc.8b05047

Yu, M. B., Firek, A., and Langridge, W. (2018). Predicting Methotrexate Resistance in Rheumatoid Arthritis Patients. Inflammopharmacol 26 (3), 699-708. doi:10.1007/s10787-018-0459-Z

Zhai, K. F., Duan, H., Cui, C. Y., Cao, Y. Y., Si, J. L., Yang, H. J., et al. (2019). Liquiritin from Glycyrrhiza Uralensis Attenuating Rheumatoid Arthritis via Reducing Inflammation, Suppressing Angiogenesis, and Inhibiting MAPK Signaling Pathway. J. Agric. Food Chem. 67 (10), 2856-2864. doi:10.1021/acs.jafc.9b00185

Zhang, W., Li, F., and Gao, W. (2017). Tripterygium Wilfordii Inhibiting Angiogenesis for Rheumatoid Arthritis Treatment. J. Natl. Med. Assoc. 109 (2), 142-148. doi:10.1016/j.jnma.2017.02.007

Zhao, Y., Li, Y., Mao, S., Sun, W., and Yao, R. (2015). The Influence of Printing Parameters on Cell Survival Rate and Printability in Microextrusion-Based 3D Cell Printing Technology. Biofabrication 7 (4), 045002. doi:10.1088/1758-5090/ $7 / 4 / 045002$

Zhao, Y., Yao, R., Ouyang, L., Ding, H., Zhang, T., Zhang, K., et al. (2014). Threedimensional Printing of Hela Cells for Cervical Tumor Model In Vitro. Biofabrication 6 (3), 035001. doi:10.1088/1758-5082/6/3/035001 
Zhu, X., Li, H., Huang, L., Zhang, M., Fan, W., and Cui, L. (2020). 3D Printing Promotes the Development of Drugs. Biomed. Pharmacother. 131, 110644. doi:10.1016/j.biopha.2020.110644

Conflict of Interest: The authors declare that the research was conducted in the absence of any commercial or financial relationships that could be construed as a potential conflict of interest.

Publisher's Note: All claims expressed in this article are solely those of the authors and do not necessarily represent those of their affiliated organizations, or those of the publisher, the editors and the reviewers.
Any product that may be evaluated in this article, or claim that may be made by its manufacturer, is not guaranteed or endorsed by the publisher.

Copyright (c) 2021 Lin, Sun, Li, Yuan, Cheng, Ke, Chen, Sun, Mi and Zhang. This is an open-access article distributed under the terms of the Creative Commons Attribution License (CC BY). The use, distribution or reproduction in other forums is permitted, provided the original author(s) and the copyright owner(s) are credited and that the original publication in this journal is cited, in accordance with accepted academic practice. No use, distribution or reproduction is permitted which does not comply with these terms. 